\title{
Моделирование адсорбции нежестких органических молекул на графитированной термической саже методом Монте-Карло для предсказания их характеристик удерживания
}

\author{
Матюшин Д.Д., Буряк А.К. \\ Федеральное Государственное Бюджетное Учреждение Науки Институт физической химии \\ и электрохимии имени А.Н. Фрумкина РАН, Москва \\ Поступила в редакцию 31.01.2017 г.
}

\begin{abstract}
Был разработан новый вариант молекулярно-статистического метода для расчета констант Генри органических веществ на графитированной термической саже для нежестких молекул с учетом внутреннего вращения. Наблюдалось хорошее совпадение рассчитанных и экспериментальных констант Генри для ряда алканов, хлоралканов, простых эфиров и алкилбензолов.

Ключевые слова: метод Монте-Карло, молекулярно-статистические расчеты, графитированная термическая сажа, газовая хроматография.
\end{abstract}

\section{The Monte-Carlo molecular modeling of non-rigid organic molecules for prediction their retention properties}

\author{
Matyushin D.D., Buryak A.K. \\ Institute of Physical chemistry and Electrochemistry, RAS, Moscow
}

The Monte-Carlo molecular modeling of adsorption of organic molecules on Graphitized Thermal Carbon Black (GTCB) is provided in this work. Only that conditions are considered, where Henry's law is applicable. Modified GAFF (General Amber Force Field) force field and simplified model of molecule with rigid bond lengths and angles are used. Crowell's approach for efficient calculation of potential energy of interactions between organic molecule and graphitic layer is applied. Good agreement with experiment is demonstrated for many organic molecules: alkanes $\left(\mathrm{CH}_{4}-\mathrm{C}_{12} \mathrm{H}_{26}\right)$, chloroalkanes, alkylbenzenes, ethers. Satisfactory correlation between experimental and calculated data is achieved. New approach is compared with molecular statistical method, which is used to calculate characteristics of adsorption on GTCB. Advantage of this approach is shown. Influence of adsorption on graphitic surface at conformation of molecule is discussed. Possible effect of systematic error in experimental data is shown. All calculations are perfomed with self-written software.

Keywords: Monte-Carlo molecular modeling, graphitized thermal carbon black, gas chromatography.

\section{Введение}

Для оценки характеристик хроматографического поведения веществ на углеродных сорбентах широко применяется метод молекулярно-статистических расчетов [1]. Впервые метод разработан в 70-ые годы Киселевым и Пошкусом для варианта газовой хроматографии и графитированной термической сажи (ГТС) в качестве сор- 
бента [2]. Данный сорбент обладает однородной на атомном уровне и плоской поверхностью, взаимодействие молекул сорбата с которой относительно просто моделировать. Удерживание на ГТС чрезвычайно чувствительно к геометрии разделяемых веществ и позволяет разделять смеси изомеров, обладающих близкими физическими свойствами [3-5]. Так, например, наблюдается значительно более сильное взаимодействие пара-ди-н-бутилбензола по сравнению с пара-ди-третбутилбензолом, так как в первом случае (при оптимальном положении молекулы относительно поверхности графита) все атомы углерода находятся на близком к оптимальному расстоянии от поверхности [6].

Данный метод позволяет рассчитать константу генри $\mathrm{K}_{1}$ для адсорбции квазижестких молекул на ГТС и, соответственно, хроматографические параметры времена удерживания. Показано очень хорошее совпадение с экспериментом для целого ряда классов органических соединений. Однако в рамках этого метода молекулы рассматривались как жесткие, не имеющие конформеров. Для вращения вокруг $\sigma$-связей требуется учесть доли различных конформеров в смеси и найти $\mathrm{K}_{1}$ для каждого из них [7]. При большом числе $\sigma$-связей такое рассмотрение становится очень трудоемким.

Ранее было показано, что наблюдается сильная корреляция между результатами молекулярно-статистических расчетов и временами удерживания на популярном углеродном сорбенте «Гиперкарб» в условиях жидкостной хроматографии [8-9]. Это может иметь практическое значение при использовании этого сорбента.

Целью данной работы была разработка нового варианта метода молекулярностатистических расчетов для нежестких молекул с учетом внутреннего вращения с использованием алгоритма Метрополиса (метод Монте-Карло). В качестве пробного объекта для проверки экспериментальных методов были выбраны алканы $\left(\mathrm{C}_{4}-\mathrm{C}_{12}\right)$, алкилбензолы, простые эфиры, хлоралканы.

\section{Теоретическая часть} образом:

Константа Генри в самом общем случае может быть выражена следующим

$$
K_{1}=\frac{\mathrm{V}}{\mathrm{A}} \cdot \frac{N_{a d s}}{N_{g a s}}=\frac{V \int_{a d s} e^{\frac{-\Phi(\bar{r})}{k T}} d r}{A \int_{g a s} e^{\frac{-\Phi(r)}{k T}} d r}
$$

где $\mathrm{V}$ и А - объем газа и площадь сорбента, $\mathrm{N}_{\mathrm{abs}}$ и $\mathrm{N}_{\mathrm{gas}}$ - число молекул на поверхности и в газовой фазе соответственно. $\Phi(\vec{r})$ - потенциальная энергия молекулы для данного состояния, а интегрирование на поверхности и в газовой фазе ведется по всем возможным состояниям молекулы: по всем положениям, ориентациям в пространстве и конформациям.

Энергия взаимодействия молекулы с поверхностью графита оценивается как сумма энергий атом-атомных взаимодействий. Атом-атомные потенциалы могут быть записаны в форме Леннарда-Джонса (2) и в форме Бакингема-Корнера (3).

$$
\begin{aligned}
& w(r)=\frac{a}{r^{12}}-\frac{b}{r^{6}} \\
& w(r)=-\frac{c_{6}}{r^{6}}-\frac{c_{8}}{r^{8}}+b e^{-q r}
\end{aligned}
$$


В большинстве работ, посвященных молекулярно-статистическим, расчетам использовался потенциал в форме Бакингема-Корнера. Однако в других областях вычислительной химии чаще всего используется потенциал в форме ЛеннардДжонса. В связи с этим, имеется гораздо больше литературных данных для параметров этого потенциала. Кроме того, потенциал в этой форме предпочтителен с вычислительной точки зрения, из-за отсутствия экспоненциального слагаемого.

Для ускорения расчета применяется приближение Крауэлла [10]: поверхность графита предполагается однородной и изотропной, равномерно заполненной атомами с некоторой плотностью $\vartheta$.

$$
\begin{aligned}
& \Phi(\vec{r})=\sum_{i=0}^{i=N} \sum_{n=0}^{\infty} \int_{-\infty}^{+\infty} \int_{-\infty}^{+\infty} \frac{A_{i}}{\left(x_{i}^{2}+y_{i}^{2}+\left(z_{i}+n d\right)^{2}\right)^{6}}-\frac{B_{i}}{\left(x_{i}^{2}+y_{i}^{2}+\left(z_{i}+n d\right)^{2}\right)^{3}} d x d y= \\
& =\vartheta \pi \sum_{i=0}^{i=N} \frac{1}{2 d^{4}} A_{i} \xi\left(\frac{{ }_{i}}{d}, 4\right)-\frac{1}{2 d^{4}} B_{i} \xi\left(\frac{z_{i}}{d}, 10\right)
\end{aligned}
$$

Интегрирование ведется по площади поверхности графита. Суммирование ведется по всем $\mathrm{N}$ атомам в молекуле и по плоскостям графита (межплоскостное расстояние d). Для дальнейшего упрощения расчета колебания адсорбированной молекулы в направлении перпендикулярном слою графита считаются гармоническими вблизи минимума потенциальной энергии, равного $\Phi_{0}[2]$.

С учетом этого для жесткой трехмерной молекулы константа Генри записывается следующим образом:

$$
K_{1}=\frac{\frac{1}{4 \pi} \int_{\alpha_{1} \alpha_{2} \ldots \alpha_{n}}^{\iint} \int\left(\frac{2 k T}{\Phi_{z}^{\prime \prime}}\right)^{\frac{1}{2}} \exp \left(-\frac{\Phi_{0}+\Phi_{\text {int }}}{k T}\right) \sin \Theta d \Theta d \Psi d \alpha_{1} d \alpha_{2} \ldots d \alpha_{n}}{\int_{\alpha_{1} \alpha_{2} \ldots \alpha_{n}} \exp \left(-\frac{\Phi_{\text {int }}}{k T}\right) d \alpha_{1} d \alpha_{2} \ldots d \alpha_{n}}
$$

где T - температура; $\mathrm{k}$ - постоянная Больцмана; $\Theta$ и $\psi$ - углы Эйлера, определяющие ориентацию молекулы относительно поверхности адсорбента, $\alpha_{\mathrm{i}}-$ i-ый угол вращения, a $\Phi_{\text {int }}-$ внутренняя энергия данной конформации. Для оценки внутренней энергии молекулы $\Phi_{\text {int }}$ использовалось приближение из работы [11].

\section{Эксперимент}

Расчет константы Генри на ГТС проводился в соответствии с формулами (4), (5). Параметры взаимодействий для оценки внутримолекулярных взаимодействий брались из разработанного для методов молекулярной динамики набора GAFF (General Amber Force Field) [11]. Выбор данного набора обусловлен хорошим соответствием предсказанных барьеров вращения с экспериментальными данными и возможностью, в дальнейшем, использовать те же методы для моделирования адсорбции на сорбенте «Гиперкарб» в условиях жидкостной хроматографии.

Параметры потенциала Леннарда-Джонса для взаимодействия с атомами графита отличаются от таковых для взаимодействия с атомом углерода в органической молекуле. Эти параметры оценивались в различных работах [12-14], во всех случаях абсолютная величина в минимуме потенциальной энергии меньше примерно на 10$20 \%$ в случае графита. Нами были предварительно подобраны следующие значения минимума энергии и Ван-дер-Ваальсова радиуса.

$$
{ }^{\varepsilon_{C, \text { графит }}}=0.0702 \text { ккал } / \text { моль; } \quad r_{C \text {,графит }}=1.908 \mathrm{~A}
$$

Для каждого типа атома молекулы минимум потенциальной энергии принимался равным в соответствии с правилом Бертло. Значения и радиусов атомов бра- 
лись так же из набора GAFF. Расстояние между плоскостями графита принималось равным 0.356 нм, плотность атомов на поверхности графита 0.382 нм $^{-2}$. Начальная геометрия молекулы оптимизировалась путем минимизации с помощью метода сопряженных градиентов внутренней энергии, оцененной в соответствии с работой [11].

Интегрирование по углам внутреннего вращения производилось с помощью метода Монте-Карло, в соответствии с алгоритмом Метрополиса. На каждом шаге алгоритма производился поворот вокруг одной из связей на произвольный угол. Валентные углы и длины связей предполагались неизменными при изменении конформации. Вычисления продолжались до достижения точности \pm 0.01 для логарифма константы Генри $\left(\mathrm{cm}^{3} / \mathrm{m}^{2}\right)$. Все вычисления проводились с помощью специально разработанной нами программы. Экспериментальные данные по константам Генри для сорбции алканов, хлоралканов и простых эфиров на ГТС были взяты из работы [2], для н-алкилбензолов из работ [2] и [15].

\section{Обсуждение результатов}

Внутреннее вращение существенно влияет на адсорбцию на ГТС. Для многих молекул наиболее энергетически выгодная конформация в свободном и в адсорбированном виде существенно отличаются. Так, например, было показано, что для этилбензола в свободном состоянии наиболее выгодной является конформация, в которой этильная группа находится в плоскости, перпендикулярной плоскости бензольного кольца.

На рис. 1 показаны оптимальные конформации н-бутилбензола и н-додекана в свободном и адсорбированном состоянии. В свободном состоянии молекула изогнута так, что разные части молекулы сближены друг с другом за счет сил Ван-дерВаальса. Константы Генри рассчитанные для различных конформаций с использованием модели жесткой молекулы отличаются во много раз. Для н-бутилбензола разница внутренних энергий для конформаций (а) и (б) 8.5 кДж/моль, для н-додекана 1.5 кДж/моль. Разница энергий взаимодействия с графитом (при оптимальном положении молекулы относительно поверхности графита) -12.3 кДж/моль и -9.9 кДж/моль.

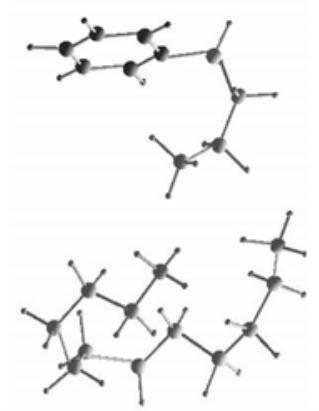

a)

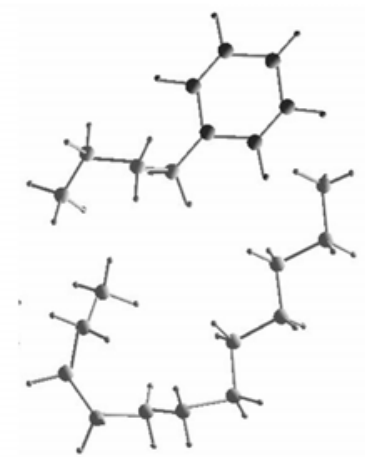

6)

Рис. 1. Оптимальные конформации н-бутилбензола и н-додекана в свободном (а) и адсорбированном на ГТС (б) состоянии.

На рисунке 2а приведена корреляционная зависимость экспериментально измеренных и рассчитанных констант Генри для ряда органических молекул для $300^{\circ} \mathrm{K}$. Несмотря на то, что измерения реально производились при иных температу- 
pax, сравнения констант Генри целесообразно производить, приведя их к одной температуре, в связи с приблизительно линейной зависимостью $\mathrm{K}_{1}$ от $1 / \mathrm{T}$ в широком диапазоне температур. Рассчитанные значения приведены в таблице 1 . Полученная корреляционная зависимость описывается уравнением $\operatorname{lnK}_{1}$ (эксп.) $=\operatorname{lnK}_{1}$ (расч.)·0.9555-0.2543 $\left(\mathrm{R}^{2}=0.99\right)$.

a)

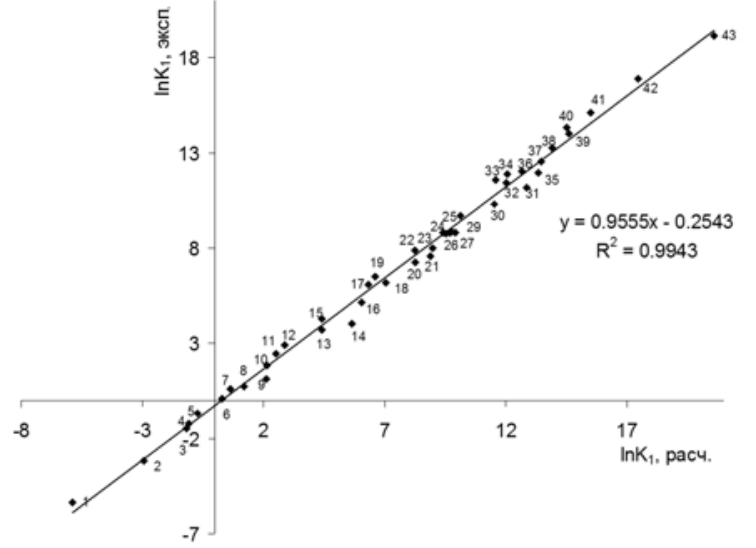

6)
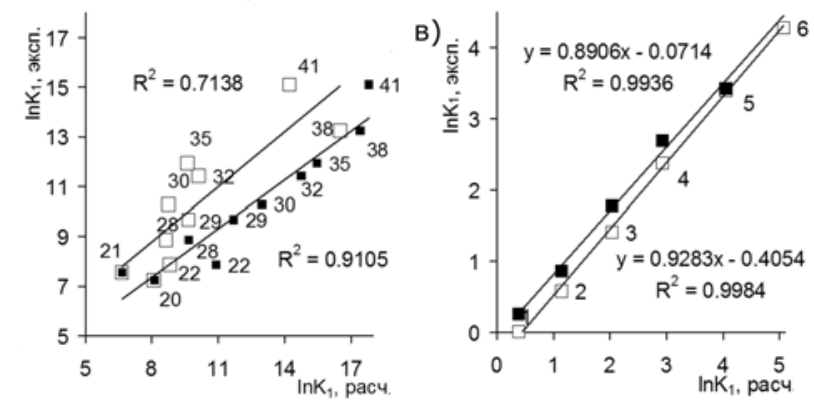

Рис. 2. Корреляционные зависимости экспериментально измеренных и рассчитанных с учетом внутреннего вращения (a, в) и без учета (б) логарифмов констант Генри, см ${ }^{3} / \mathrm{m}^{2}$. Подписи точек для рисунков а), б) даны в соответствии с таблицей 1 , на рисунке в) цифрами обозначены: 1 - толуол, 2 - этилбензол, 3 - н-пропилбензол, 4 - н-бутилбензол, 5 - н-пентилбензол, 6 - н-гексилбензол.

Таблица 1. Рассчитанные значения констант Генри $\ln \mathrm{K}_{1} \mathrm{~cm}^{3} / \mathrm{M}^{2}$ для адсорбции органических веществ на ГТС при $300^{\circ} \mathrm{K}$.

\begin{tabular}{|c|c|c|c|c|c|}
\hline $\mathrm{N}$ & вещество & $\begin{array}{r}\operatorname{lnK} K_{1} \\
\mathrm{~cm}^{3} / \mathrm{m}^{2}\end{array}$ & $\mathrm{~N}$ & вещество & $\begin{array}{r}\operatorname{lnK} K_{1} \\
\mathrm{~cm}^{3} / \mathrm{m}^{2}\end{array}$ \\
\hline 1 & 2 & 3 & 4 & 5 & 6 \\
\hline 1 & Метан & -5.88 & 23 & Диизобутиловый эфир & 8.99 \\
\hline 2 & Этан & -2.95 & 24 & Мета-ксилол & 9.52 \\
\hline 3 & Пропан & -1.18 & 25 & Пара-ксилол & 9.4 \\
\hline 4 & Бутан & -1.08 & 26 & Орто-ксилол & 9.9 \\
\hline 5 & Диметиловый эфир & -0.72 & 27 & Ди-н-бутиловый эфир & 9.68 \\
\hline 6 & Хлорэтан & 0.3 & 28 & н-Пропилбензол & 9.77 \\
\hline 7 & Изобутан & 0.63 & 29 & н-Нонан & 10.13 \\
\hline 8 & н-Бутан & 1.2 & 30 & н-Бутилбензол & 11.53 \\
\hline 9 & Неопентан & 2.11 & 31 & Диизоамиловый эфир & 12.86 \\
\hline 10 & Диэтиловый эфир & 2.14 & 32 & н-Декан & 12.03 \\
\hline 11 & н-Пентан & 2.52 & 33 & 1,3,5-Триметилбензол & 11.58 \\
\hline 12 & 1-Хлорбутан & 2.87 & 34 & 1,3,4-Триметилбензол & 12.07 \\
\hline 13 & Бензол & 4.42 & 35 & н-Пентилбензол & 13.35 \\
\hline 14 & Диизопропиловый эфир & 5.66 & 36 & 123-Триметилбензол & 12.66 \\
\hline 15 & н-Гексан & 4.42 & 37 & Ди-н-амиловый эфир & 13.47 \\
\hline
\end{tabular}




\begin{tabular}{|c|c|c|c|c|c|}
\hline 1 & 2 & 3 & 4 & 5 & 6 \\
\hline 16 & Ди-н-пропиловый эфир & 6.06 & 38 & н-Ундекан & 13.9 \\
\hline 17 & н-Гептан & 6.33 & 39 & 1,2,4,5-тетраметилбензол & 14.6 \\
\hline 18 & Толуол & 7.05 & 40 & 1,2,3,5-тетраметилбензол & 14.51 \\
\hline 19 & 1-Хлоргексан & 6.6 & 41 & н-додекан & 15.51 \\
\hline 20 & Этилбензол & 8.27 & 42 & Пентаметилбензол & 17.47 \\
\hline 21 & Изопропилбензол & 8.89 & 43 & Гексаметилбензол & 20.6 \\
\hline 22 & н-Октан & 8.24 & & & \\
\hline
\end{tabular}

Для сравнения был выполнен расчет константы Генри для ряда моноалкилбензолов и н-алканов без учета внутреннего вращения. Результаты данного расчета приведены на рис. 2б. Расчет выполнялся для конформаций оптимальных в свободном (контурные маркеры) и в адсорбированном (сплошные маркеры) состоянии. Полученные данные не показывают достаточно хорошего совпадения с экспериментально измеренными константами Генри. Величина квадрата коэффициента корреляции составляет 0.91 и 0.71 для конформаций, оптимальных в свободном и в адсорбированном виде соответственно. Таким образом, показано, что расчеты по методу Монте-Карло с учетом внутреннего вращения позволяют удовлетворительно предсказать константу Генри, а значит и время удерживания, в то время как применявшийся ранее молекулярно-статистический метод не дает приемлемого результата для таких нежестких молекул.

Было проведено сравнение расчетных констант Генри с экспериментально измеренными, взятыми из двух различных источников. На рис. 2в показаны аналогичные корреляционные зависимости для н-алкилбензолов рассчитанные для температуры $\mathrm{T}=450^{\circ} \mathrm{K}$.

Экспериментальные данные взяты из источников [2] (сплошные маркеры) и [15] (контурные маркеры). В обоих случаях наблюдается линейная зависимость между измеренными и рассчитанными значениями, однако параметры аппроксимации - угловой коэффициент и точка пересечения с осью ОY - значительно отличается, что свидетельствует о систематической ошибке измерения как минимум в одном из источников. Полученные корреляционные зависимости описываются уравнениями $\operatorname{lnK}_{1}$ (эксп. $)=\ln K_{1}$ (расч.) $0.8906-0.0714\left(\mathrm{R}^{2}=0.9936\right)-$ для данных из работы [2]. $\operatorname{lnK}_{1}$ (эксп. $)=\operatorname{lnK}_{1}$ (расч.) $0.9283-0.4054\left(\mathrm{R}^{2}=0.9984\right)$ - для данных из работы [15].

\section{Заключение}

Был разработан новый вариант метода молекулярно-статистических расчетов для нежестких молекул с использованием алгоритма Метрополиса. Этим методом было проведено моделирование адсорбции ряда органических молекул на ГТС. Вычисления основывались на приближении, ранее использованном для молекулярностатистических расчетов. Было показано, что данный метод позволяет получить удовлетворительные предсказания для констант Генри адсорбции на ГТС для молекул, подверженных свободному вращению вокруг $\sigma$-связей, в то время как метод молекулярно-статистических расчетов, в соответствии с которым молекулы считаются жесткими, не давал удовлетворительных результатов для таких соединений. Так же была показана возможность применения потенциала в форме Леннард-Джонса для моделирования адсорбции на ГТС с использованием параметров потенциала, полученных с помощью правила смешивания Бертло. 


\section{Список литературы}

1. Buryak A.K. // Russ Chem Rev. 2002. Vol. 71(8). pp. 695-706.

2. Авгуль Н.Н., Киселев А.В., Пошкус Д.П. Адсорбция газов и паров на однородных поверхностях. М.: Химия. 1975. 384 с.

3. Kiselev A.V., Nazarova V.I., Shcherbakova K.D. // Journal of Chromatography A. 1984. Vol. 292. No 1. pp. 97-103.

4. Kiselev A.V., Kulikov N.S., Curthoys G. // Chromatographia. 1984. Vol. 18. Issue 6. pp. 297-304.

5. Engewald W., Pörschmann J., Welsch T. // Chromatographia. 1990. Vol. 30. Issue 9, pp. 537-542.

6. Engewald W., Wennrich L., Pörschmann J. // Chromatographia. 1978. Vol. 11. No 8. pp. 434-439.

7. Буряк А.К., Гарькин В.П., Редькин Н.А., Сердюк Т.М. и др. Молекулярностатистические расчеты термодинамических характеристик адсорбции изомеров: учебное пособие. Самара: «Универс групп». 2008. $142 \mathrm{c}$.

8. Милюшкин А.Л., Буряк А.К. // Сорбиионные и хроматографические прочессы. 2015. T. 15. № 3. C. 379-389.

\section{References}

1. Buryak A.K., Russ Chem Rev., 2002,Vol. 71(8), pp. 695-706.

2. Avgul' N.N., Kiselev A.V., Poshkus D.P. Adsorbtsiya gazov i parov na odnorodnykh poverkhnostyakh (Adsorption of gases and vapors on homogeneous surfaces), M., Khimiya, 1975, pp. 384.

3. Kiselev A.V., Nazarova V.I., Shcherbakova K.D., Journal of Chromatography A, 1984, Vol. 292., No 1, pp. 97-103.

4. Kiselev A.V., Kulikov N.S., Curthoys G., Chromatographia, 1984, Vol. 18, No 6, pp. 297-304.

5. Engewald W., Pörschmann J., Welsch T., Chromatographia. 1990, Vol. 30, No 9, pp. 537-542.

6. Engewald W., Wennrich L., Pörschmann J., Chromatographia, 1978, Vol. 11, No 8, pp. 434-439.

7. Buryak A.K., Gar'kin V.P., Red'kin N.A., Serdyuk T.M. et al., Molekulyarnostatisticheskie raschety termodinamicheskikh kharakteristik adsorbtsii izomerov: uchebnoe
9. Kuznetsova E.S., Buryak A.K. // Colloids and Surfaces A: Physicochem. Eng. Aspects. 2011. Vol. 383. No 1-3. pp. 73-79.

10. Crowell A.D., Steele R.B. // J. Chem. Phys. 1961. Vol. 34. pp. 1347-1349.

11. Wang J., Wolf R M., Caldwell J.W., Kollman P.A. et al. // Journal of Computational Chemistry. 2004. Vol. 25. pp. 1157-1174.

12. Leroy F., Liu S., Zhang J. // The Journal of Physical Chemistry C. 2015. Vol.119. No 51. pp. 28470-28481.

13. Werder T., Walther J.H., Jaffe R.L., Halicioglu T. et al. // J. Phys. Chem. B. 2003. Vol. 107. No 6. pp. 1345-1352.

14. Wu. Y.B., Aluru N.R. // J. Phys. Chem. B. 2013. Vol. 117. No 29. pp. 88028813.

15. Kalashnikova E.V., Kiselev A.V., Petrova R.S., Shcherbakova K.D. et al. // Chromatographia. 1979. Vol. 12 No 12. pp. 799-802.

posobie (Molecular-statistical calculations of thermodynamic characteristics of adsorption of isomers: study book), Samara, Univers grupp, 2008, pp. 142.

8. Milyushkin A.L., Buryak A.K., Sorbtsionnye $i$ khromatograficheskie protsessy, 2015, Vol. 15, No 3, pp. 379-389.

9. Kuznetsova E.S., Buryak A.K., Colloids and Surfaces A: Physicochem. Eng. Aspects, 2011, Vol. 383, No 1-3, pp. 73-79.

10. Crowell A.D., Steele R.B., J. Chem. Phys., 1961, Vol. 34, pp. 1347-1349.

11. Wang J., Wolf R M., Caldwell J.W., Kollman P.A., et al., Journal of Computational Chemistry, 2004, Vol. 25, pp. 1157-1174.

12. Leroy F., Liu S., Zhang J., The Journal of Physical Chemistry C, 2015, Vol. 119, No 51, pp. 28470-28481.

13. Werder T., Walther J.H., Jaffe R.L., Halicioglu T. et al., J. Phys. Chem. B, 2003, Vol. 107, No 6, pp. 1345-1352.

14. Wu. Y.B., Aluru N.R., J. Phys. Chem. B, 2013, Vol. 117, No 29, pp. 8802-8813. 
15. Kalashnikova E.V., Kiselev A.V., Petrova R.S., Shcherbakova K.D. et al., Chroma-

Матюшин Дмитрий Дмитриевич - старший лаборант. Институт физической химии и электрохимии имени А.Н. Фрумкина РАН, Москва

Буряк Алексей Константинович - заведующий лабораторией физико-химических основ хроматографии и хромато-массспектрометрии, проф, д.Х.н. Институт физической химии и электрохимии имени А.Н. Фрумкина РАН, Москва tographia, 1979, Vol. 12, No 12, pp. 799-802.

Matyushin Dmitriy D. - Senior Assistant, A.N.Frumkin Institute of Physical Chemistry and Electrochemistry, Russian Academy of Sciences, Moscow. E-mail: dm.matiushin@mail.ru

Buryak Alexey K. - prof., grand $\mathrm{PhD}$ (chemistry), laboratory of physical-chemical basics of chromatography and chromato-mass-spectrometry Institute of Physical chemistry and electrochemistry, Moscow 